\title{
¿Quién estudia en la universidad? La dimensión social de la universidad española en la segunda década del siglo XXI
} Who Studies at University? The Social Dimension of the Spanish University in the $2^{\text {nd }}$ decade of the $21^{\text {st }}$ Century

\author{
Damián Herrera Cuesta'
}

\section{Resumen}

En la Cumbre de Gotemburgo, celebrada el 17 de noviembre de 2017, fue proclamado por los estados miembros de la Unión Europea, el Pilar Europeo de los Derechos Sociales, documento con el que se quiere materializar el deseo colectivo de trabajar juntos por una Europa más social. Este artículo aborda el estudio sobre el grado de «Equidad participativa» del sistema universitario en España, a partir de las condiciones socioeconómicas y educativas de los jóvenes estudiantes de nuevo ingreso. Para su análisis, se han comparado la ocupación y los estudios de los progenitores, con la población general de edad equivalente (convencionalmente entre 40 y 59 años), e igual posición ocupacional y nivel de estudios alcanzado. Los resultados obtenidos, evidencian la existencia de una infrarrepresentación severa del estudiantado con bajo Capital Educativo, así como también del estudiantado con bajo Capital Socioeconómico. En el curso 2015/16, el porcentaje de estudiantes universitarios de nuevo ingreso, cuyos progenitores se encontraban ocupados en empleos no cualificados y elementales, apenas representaban el 9,12\% y el 7,62\% (padre y madre, respectivamente), del estudiantado universitario en España.

\section{Palabras claves}

Educación superior, dimensión social, capital educativo, capital socioeconómico.

\section{Abstract}

At the Göteborg Summit, held on 17 November 2017, the European Pillar of Social Rights was proclaimed by the Member States of the Union, a document with which the collective desire to work together for a more social Europe is to materialize. This article deals with the study on the degree of «Participative Equity» of the university system in Spain based on the socioeconomic and educational conditions of the young new students. For their analysis, the occupation and the studies of the parents of the new students with the occupational position and the studies of the general population with an age equivalent to those of the former (conventionally between 40 and 59 years old) have been compared. The results obtained show the existence of a severe underrepresentation not only of students with low educational, also of students with low Socioeconomic Capital. The percentage of new university students whose parents were employed in unskilled and elementary jobs represented barely $9.12 \%$ and $7.62 \%$ (father and mother, respectively) of the university students in the 2015/16 academic year.

\section{Keywords}

Higher education, social dimension, educational capital, socioeconomic capital. 


\section{Introducción. El concepto de Dimensión Social de la Educación Superior (DSES).}

En el marco del Espacio Europeo de Educación Superior (EEES), el concepto de Dimensión Social de la Educación Superior (DSES), hace referencia a la composición social de la matrícula universitaria y los factores que la determinan (Ariño, 2014). Aparece por primera vez ligado a las políticas educativas de la UE, durante la conferencia de Londres de 2007: «La Educación Superior debe desempeñar un papel importante en el fomento de la cohesión social, la reducción de las desigualdades y el aumento del nivel de conocimientos, habilidades y competencias en la sociedad». (Comunicado de Londres, 2007: 4).

Es a partir de este momento, cuando la Dimensión Social de la Educación Superior adquiere por primera vez una plasmación significativa en las políticas europeas (Ariño \& Llopis, 2011). El acceso a la Universidad deja de considerarse una mera cuestión de números, para convertirse en un elemento clave en el desarrollo del sistema, razón por la que se empiezan considerar relevantes las acciones destinadas a eliminar las barreras que impiden a la población acceder a la Educación Superior.

Al considerar que vivimos en un entorno económico y social, en el que cada vez cobran más importancia las habilidades y las competencias adquiridas a través de la Educación Superior, la eliminación de las barreras que constriñen las oportunidades de acceso a la misma, pasa a constituirse en un imperativo necesario para el progreso social, cuyo logro ha de alcanzarse a través de políticas educativas que faciliten la «igualdad de oportunidades de acceso a una educación de calidad, así como de igualdad en el trato, incluyendo la adaptación de la educación a las necesidades de los individuos», de modo que, los sistemas de educación y formación equitativos, «estén dirigidos a proporcionar oportunidades de acceso, trato y resultados que sean independientes del nivel socioeconómico y otros factores que puedan conducir a una desventaja educativa» (COM, 2010, del 11 de mayo).

Esta elección de objetivos exhibe una tendencia general a enfatizar la importancia de la equidad en la Educación Superior ${ }^{2}$, sin embargo, y a pesar de presentarse como un eje esencial en la creación y desarrollo del EEES, son numerosos los informes que señalan que no se viene haciendo todo lo necesario para aumentar la participación social en el sentido indicado. En Europa, el incremento de la tasa de matriculación no ha ido acompañado de una mejora en las pautas de acceso de los colectivos más vulnerables.

Según el informe, «Equity in Education» (OCDE, 2018), como promedio entre los 33 países participantes en la encuesta PIAAC ${ }^{3}$, los adultos con al menos un padre con educación superior tienen onces veces más probabilidades de completar la educación terciaria que los adultos cuyos padres no han completado la escuela secundaria superior. En el marco de la Unión Europea, la encuesta «Social Inclusion Monitor Europe (SIM)» (Bertelsmann Stiftung, 2017)4 , realizada durante 2016, logró reunir la opinión de mil expertos europeos acerca de cómo habían evolucionado, en sus respectivos países, las reformas que tenían por objetivo prioritario la Dimensión Social en los sistemas de Educación Superior. El 73\% percibía la necesidad fuerte, o muy fuerte, de garantizar la igualdad de oportunidades en la Educación

2 Siguiendo a Ariño (2011), los conceptos de participación y de equidad participativa deben entenderse como traslación al ámbito universitario de los conceptos desarrollados en el ámbito de la teoría política, de participación política, participación ciudadana y «Equidad participativa, es decir, distribución equitativa de las oportunidades de acceso, trayectoria y resultado, según categorías y variables socioeconómicas o de otro tipo» (24).

3 Programme for the International Assessment of Adult Competencies (PIAAC).

4 El Barómetro sobre la Reforma ha sido desarrollado por Bertelsmann Stiftung en cooperación con Bernd Marin de la Oficina Europea de Consultoría Política e Investigación Social de Viena y Christian Keuschnigg de la Universidad de St. Gallen. Es parte del proyecto «Social Inclusion Monitor (SIM) Europe» de Bertelsmann Stiftung, que realiza regularmente evaluaciones de oportunidades para la participación social y las políticas de reforma en los 28 estados miembros de la Unión Europea. 
Superior. De hecho, con la excepción de algunos países del este de Europa (Bulgaria, Estonia, Polonia y Eslovaquia) y del norte (Dinamarca), en la mayoría de los países, entre el 60 y el 90 por ciento de estos expertos, están de acuerdo en la necesidad de intensificar las reformas dirigidas a reducir la desigualdad de oportunidades en la Educación terciaria.

En el mismo sentido, el informe sobre la implantación del proceso de Bolonia, elaborado a instancias de la Comisión, resulta igualmente concluyente. A pesar del compromiso firmado en el Comunicado de Nueva Lovaina (2009), cuya finalidad era aumentar la participación en la Educación Superior de la población general, y la de los grupos infrarrepresentados en particular, menos del $20 \%$ de los sistemas universitarios han definido objetivos respecto a los grupos infrarrepresentados. Los objetivos nacionales se vuelcan en aumentar los esfuerzos por aumentar la participación en general, si bien, «no obstante, resulta difícil determinar si un incremento global en las cifras de participación traerá consigo una composición más equilibrada de la población estudiantil» (EACEA/Eurydice, 2015:150).

Las críticas más relevantes, ponen en cuestión la racionalidad que subyace al planteamiento general que se hace desde las instituciones europeas, sobre la problemática en torno a la Dimensión Social del Sistema Educativo en general, y, en particular, del sistema universitario. Desde este enfoque crítico, se cuestiona que las instituciones europeas tengan en cuenta, realmente, las desigualdades estructurales preexistentes a la hora de elaborar sus propuestas de acción a los estados miembros. Autores como Ariño $(2011,2014)$ advierten, por ejemplo, sobre las limitaciones teóricas que enmarcan el concepto de «equidad participativa» en la Educación Superior. Un concepto que se fundamenta en el supuesto liberal tradicional, sobre la distribución aleatoria en la sociedad de las capacidades (intelectuales) «innatas» de los individuos. Bajo esta premisa, lo que se propone con el concepto de «equidad participativa», es un ajuste proporcional de la composición social de las matriculaciones universitarias con respecto a la diversidad social existente, fundamentada ésta en las diferencias biológicas y culturales, dejando en un segundo plano las diferencias socioeconómicas existente entre los individuos (Ariño, A. 2011). Estudios recientes como los de Nielsen y Roos (2015), o Jerrim et al.,(2014), han observado que la genética juega un papel menor en la explicación de los logros educativos y, por lo tanto, no puede explicar los resultados desiguales.

En este trabajo adoptamos la perspectiva de la democratización, analizamos el grado en que los bienes educativos, en este caso los relacionados con la Educación Superior, se redistribuyen socialmente, a la vez que asumimos, como evidencia empírica, que el desarrollo de las capacidades intelectuales y las competencias para el esfuerzo personal, son modelados, desde edades tempranas, a través de procesos de socialización fuertemente condicionados por los recursos económicos y culturales disponibles por las familias (Ariño, 2014). El propósito de este trabajo es, por tanto, estudiar el nivel de inclusión de la universidad española respecto de las condiciones socioeconómicas y culturales de los estudiantes. Para medir el grado de equidad en el acceso adoptamos un procedimiento similar al utilizado por «ECoVipen 2012» (2014). Comparamos el porcentaje de estudiantes cuyos padres han alcanzado un determinado nivel educativo, con el porcentaje de adultos que tienen la misma edad y nivel educativo en la población general. Para ello, cruzamos los datos publicados por el Ministerio de Educación, Cultura y Deporte (MECD), sobre las características sociodemográficas de los estudiantes de nuevo ingreso en el curso 2015/2016, con los datos publicados por el Instituto Nacional de Estadística (INE) sobre grupos de población con edades comprendidas entre los 40 y los 60 años (edades consideradas equivalentes a las de los progenitores de los estudiantes), según el nivel de educación alcanzado, la ocupación y el sexo. 


\section{Evolución de las tasas de matriculación universitaria en España según el Capital Educativo familiar del estudiantado. Periodo 2002-20155.}

El estudio de los contextos sociales en los que viven los estudiantes que acceden a la Universidad, ha venido siendo, tradicionalmente, objeto de interés para la investigación sociológica que estudia la relación entre las desigualdades estructurales, el Sistema Educativo y la movilidad social. También, como no podría ser de otro modo, debido a la extensión de la sociedad del conocimiento en el capitalismo de tercera generación, y con ello, la existencia de una cada vez mayor heterogeneidad del estudiantado universitario, las condiciones de entrada y permanencia de los estudiantes en la Universidad, ha sido asumida como una cuestión estratégica en materia de política educativa por los gobiernos europeos. Saber si las desigualdades socioeconómicas y culturales inciden en la entrada o en el rendimiento académico, o si la educación realmente contribuye a la igualdad de oportunidades, y cómo, son cuestiones que, como hemos expuesto en la introducción, están presentes en el debate político europeo.

La influencia del origen familiar sobre la trayectoria educativa de los estudiantes es un elemento clave para estudiar la movilidad social (Carabaña \& Rivière, 2017; Ariño, A. 2014). El nivel socioeconómico y educativo de los padres, tiene un impacto determinante en la probabilidad de que los hijos accedan a estudios superiores y los completen (Rahona, 2008). Partiendo de estas premisas, antes de pasar a estudiar el grado de equidad en la universidad española actual, hemos revisado la composición del estudiantado universitario durante el periodo 2002-2015, teniendo en cuenta el nivel de formación alcanzado por los progenitores de los estudiantes.

Basándonos en datos recogidos de los informes publicados por el MECD a lo largo del periodo, todo parece indicar que, efectivamente, dada la alta proporción de estudiantes con bajo Capital Educativo familiar que asiste a la Universidad, podemos decir que el Sistema Educativo en España, ha mantenido un nivel inclusivo aceptable hasta el curso 2007/08, momento en el que comienza a descender bruscamente su número. La proporción de estudiantes universitarios, cuyo padre posee un nivel educativo inferior o igual a la primera secundaria, desciende del $40 \%$ al 25\%, entre 2007 y 2015 (Véase gráfico 1), y en el caso de aquellos cuya madre no supera este nivel educativo, lo hace del $46 \%$ al $26 \%$ (Véase gráfico 2).

5 Para la elaboración del análisis longitudinal previo que presentamos en este epígrafe, hemos utilizado los datos publicados en los informes anuales elaborados por el MECD sobre las características sociodemográficas de los estudiantes universitarios. Desgraciadamente, estos datos no han sido publicados de forma continuada año a año, entre 2002 y 2012, por este motivo, en los gráficos 1 y 2, no aparecen los datos correspondientes a los años 2009 y 2010 . Solo a partir del curso 2013/2014, los datos de acceso a la Universidad aparecen publicados siguiendo pautas estables, asumiendo los acuerdos europeos, lo que nos ha permitido acometer el análisis de la composición social del sistema, cuyos resultados mostramos en los siguientes epígrafes. 


\section{Gráfico 1. Tasa de matriculación universitaria en España según nivel de estudios del padre (2002-2015)}

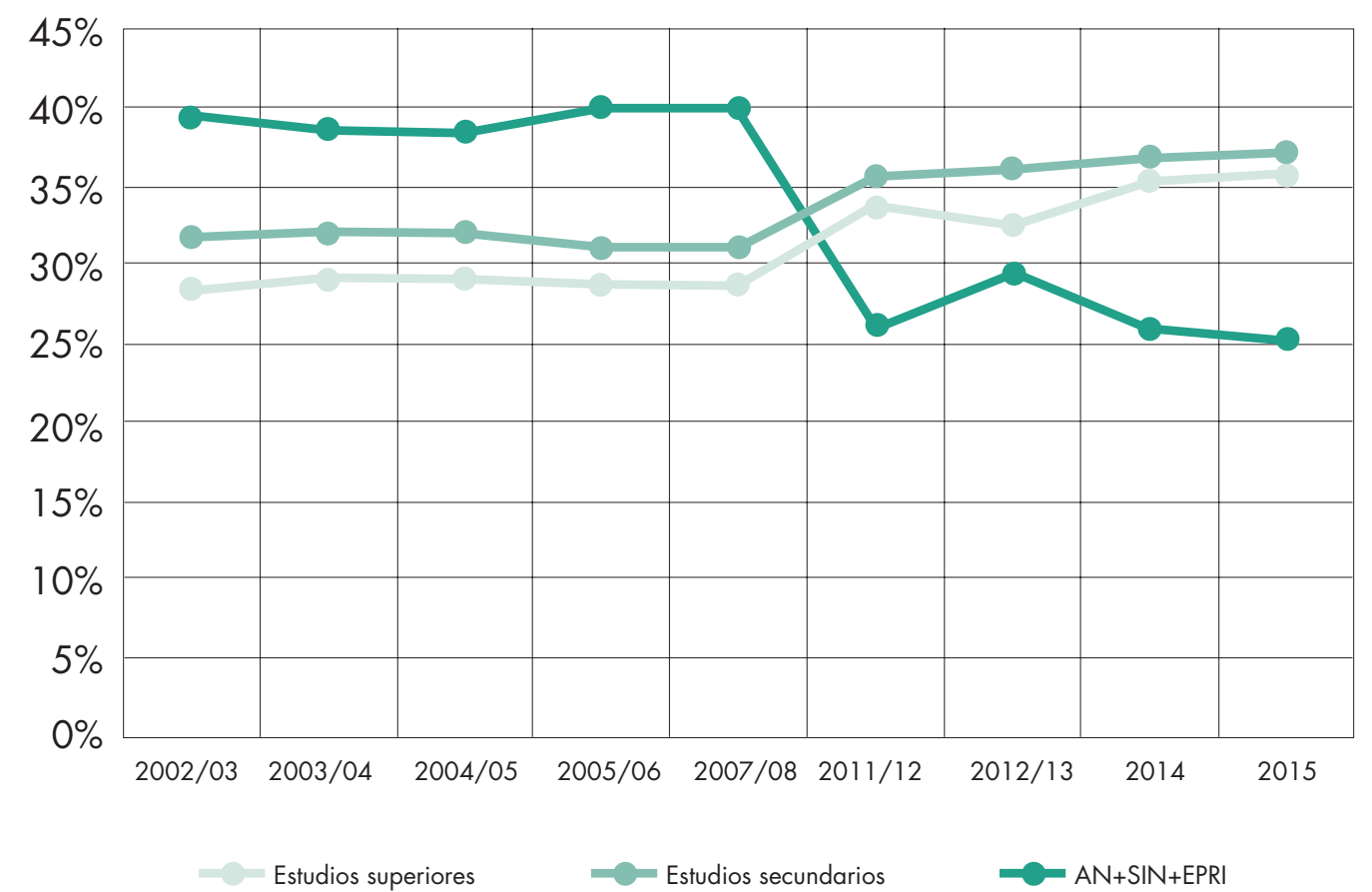

Fuente: elaboración propia a partir de datos estadísticos del MECD, varios años.

Por el contrario, aquellos estudiantes cuyos progenitores poseen estudios superiores, y aquellos que poseen hasta secundaria, o post secundaria no terciaria, mantienen una presencia proporcional regular hasta, precisamente, los cursos posteriores a 2007/2008, cuando, aquellos cuyo padre ha alcanzado un nivel educativo intermedio o superior, experimentan un incremento de 5 puntos, siendo todavía mayor este incremento cuando es la madre quien posee dicho nivel de estudios, entre 5 y 10 puntos.

\section{Gráfico 2. Tasa de matriculación universitaria en España según estudios de la madre (2002-2015)}

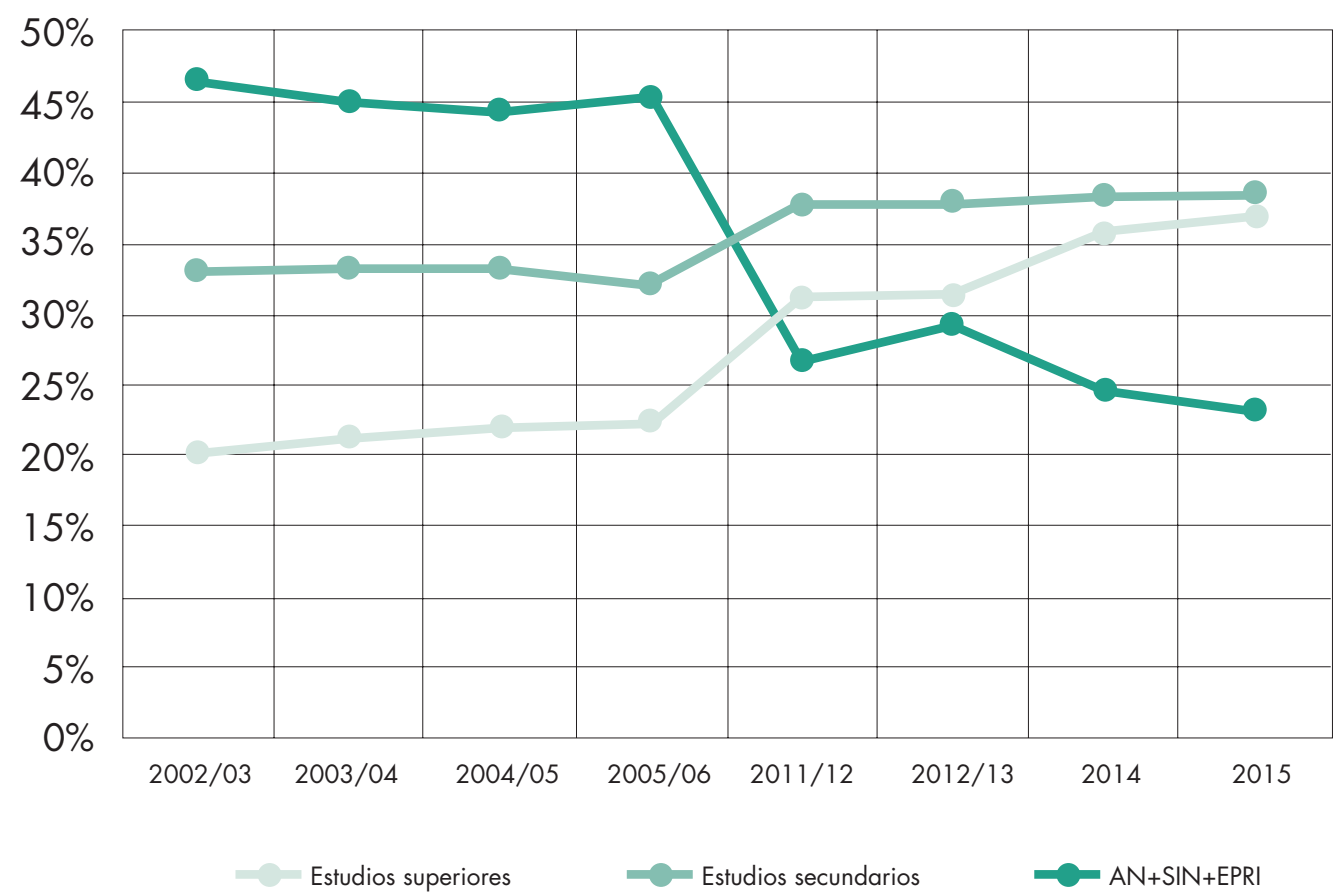

Fuente: elaboración propia a partir de datos estadísticos del MECD, varios años. 
Por tanto, podemos pensar, al menos en lo que concierne a los cursos posteriores a 2007/08, que las diferentes condiciones socioeconómicas con que las familias españolas tuvieron que hacer frente a los cambios que se produjeron en las condiciones del contexto (caída de la demanda laboral con la crisis económica, incremento de los costes directos de matrícula, políticas de becas restrictivas, etc.), guarda alguna relación con el dramático descenso de estudiantes universitarios procedentes de familias con bajo Capital Educativo, a partir de entonces. En los dos epígrafes siguientes, analizamos la magnitud y profundidad de esta tendencia, integrando en el análisis, además del Capital educativo, el Capital socioeconómico de los estudiantes.

\section{Metodología}

En el presente artículo se aborda la influencia de la posición socioeconómica de las familias en el acceso a la educación de los estudiantes universitarios en España considerando dos variables clave: la ocupación de sus progenitores y sus respectivos niveles educativos. Específicamente, hemos investigado si existe, en la Universidad española, una infrarrepresentación de estudiantes con bajos capitales socioeconómicos y educativos. Para este objetivo, comparamos el nivel educativo y el nivel de ingresos de los progenitores de las personas matriculadas en la universidad, con el nivel educativo y ocupacional de las personas que en ese momento en la estructura social tienen una edad equivalente a la de los progenitores (convencionalmente entre 40 y 59 años). Realizamos esta comparación apoyándonos en los datos que ofrecen las estadísticas universitarias del MECD; así como, en los que proporciona la Encuesta de Población Activa $(\mathrm{EPA})^{6}$.

En el análisis de la participación real de los estudiantes con diferente Capital Educativo familiar, utilizamos como referencia el nivel educativo de los padres según la clasificación CNED-2014. Posteriormente, comparamos la representación de los diferentes grupos y niveles educativos, con el conjunto de población adulta de la misma edad (entre 40 y 59 años) e idéntico nivel educativo. Los códigos de la CNED2014, , , 1 y 2 (Sin estudios, estudios de primaria y primera secundaria) los clasificamos como nivel educativo bajo; los códigos 3 y 4 (Educación secundaria y postsecundaria no terciaria) medios; y los códigos 5, 6, 7 y 8 (Educación Superior), altos.

Para determinar el peso del Capital Socioeconómico de los estudiantes en la Educación Superior para el curso 2015-2016, cruzamos los datos estadísticos del MECD sobre las características socioeconómicas de los estudiantes universitarios de nuevo ingreso, con los datos de la EPA (CNO-2011). Este procedimiento nos permite comparar la ocupación (ISCO) ${ }^{7}$ de los progenitores de los estudiantes de nuevo ingreso, con la posición ocupacional de la población general con edad equivalente a la de éstos (40 y 59 años).

Concluimos esta nota metodológica subrayando que, con el fin de poder realizar una comparativa de resultados sobre la equidad de la Universidad española entre 2011 y 2015, empleamos las referencias metodológicas utilizadas en ECoVipeu 2012. Así, la categorí«“High White collar» (ISCO 1, 2 o 3 ) agrupa las categorías (CNO-11): Directores y gerentes, Técnicos y profesionales y Técnicos de apoyo; la «Low White Collar» (ISCO 4 y 5): Empleados administrativos y de servicios; la «High Blue Collar» (ISCO 6 y 7): Trabajadores agrarios y de la industria, cualificados; y la «Low Blue Collar» (ISCO 8 y 9): Trabajadores no cualificados y Trabajadores empleados en ocupaciones elementales.

6 Se trabaja con el tercer trimestre de 2015 para la comparación con las estadísticas universitarias de acceso correspondientes al curso $2015 / 2016$.

7 ISCO (Clasificación Internacional Uniforme de Ocupaciones, por sus siglas en inglés). 


\section{Representación del Capital Educativo familiar del estudiantado de nuevo ingreso en la universidad española en la actualidad}

Tradicionalmente, la expansión de la Educación Superior en España ha sido calificada como un proceso más extensivo y relativo que democrático, debido a la infrarrepresentación en la universidad de aquellos grupos sociales con bajo capital socioeconómico y educativo (Langa, 2003; Ariño, A. 2014; Carabaña, 2016). En los gráficos que siguen (3 y 4), vemos que, al comparar el Capital Educativo de los ascendientes familiares de los estudiantes respecto del Capital Educativo de los hombres y mujeres de su generación, se hace patente, considerando esta variable, el carácter restringido del proceso de ingreso a la universidad española en la actualidad.

En el gráfico 3, observamos la existencia de sobrerrepresentación de estudiantes cuyos padres poseen un nivel educativo medio (37,88\%), o alto (36,42\%). Ambas proporciones son superiores, en relación con la proporción de hombres de su misma generación (40-60 años), y con los mismos niveles educativos (22,52\% y 31,49\% respectivamente). Por el contrario, los estudiantes cuyos padres poseen niveles bajos de educación $(23,88 \%$ ), se encuentran infrarrepresentados en relación con el porcentaje de hombres de la misma generación y con el mismo nivel de estudios que sus padres $(45,26 \%)$.

\section{Gráfico 3. Estudiantado universitario según nivel de estudios del padre/población hombres 40-60 años según nivel de educación 2015-T3}

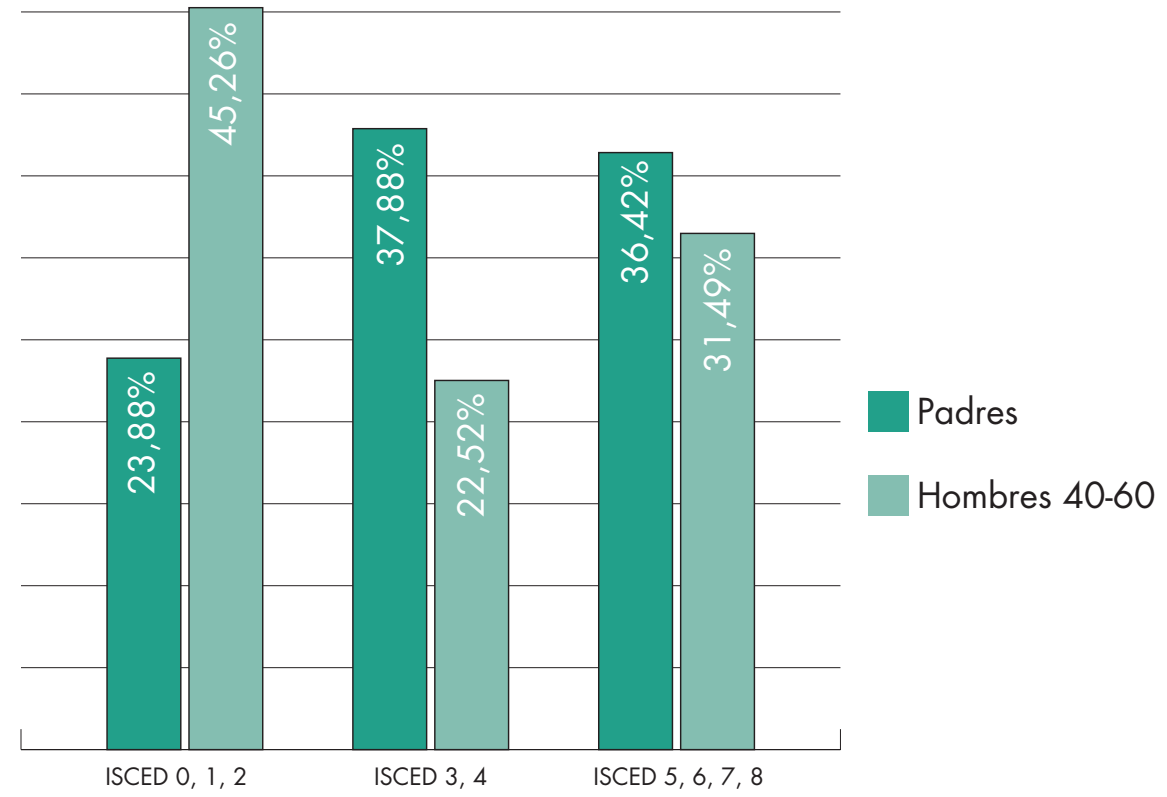

Fuente: elaboración propia a partir de datos estadísticos del MECD.

Al cuantificar a los estudiantes de nuevo ingreso según el nivel de estudios de la madre (Gráfico 4), encontramos resultados muy similares, excepto con los estudiantes cuyas madres poseen niveles educativos medios ISCED 3 y 4 (39,24\%/22,25\%). Estos estudiantes se encuentran algo más sobrerrepresentados en la Universidad. 


\section{Gráfico 4. Estudiantado universitario según nivel de estudios de la madre/población mujeres 40-60 años según nivel de educación 2015-T3}

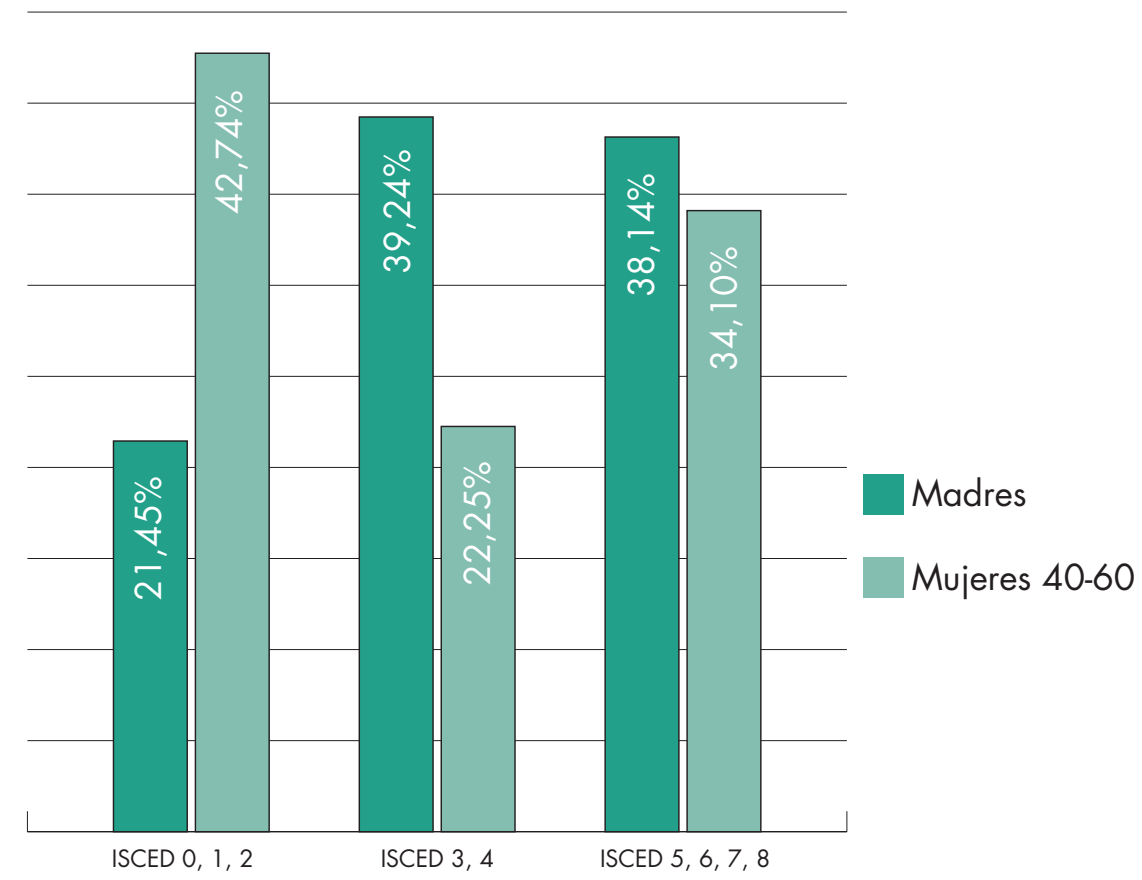

Fuente: elaboración propia a partir de datos estadísticos del MECD

En el caso de que lograsen finalizar los estudios, si sumamos los que acceden con Capital Educativo bajo (ISCED 0, 1, 2) y los que lo hacen con un Capital Educativo medio (ISCED 3 y 4) (Véase gráfico 4), el $61 \%$ de los estudiantes habrán conseguido superar el nivel educativo de la madre, y el 62\% el del padre (Gráfico 3). En consecuencia, puede decirse que la expansión de la Educación Superior en España produjo un efecto positivo en la movilidad educativa intergeneracional, uno de sus principales objetivos. Los resultados de ECoVipeu (2012) son muy similares, el 65,6\% de los estudiantes estaría en disposición de superar el nivel de estudios de la madre, y el 62,2\% el del padre (Ariño, A., Llopis, R., \& Soler, I. 2014: 53). También, para el ámbito de Cataluña, Fachelli y Planas (2011) encontraron que el porcentaje era del $70 \%$ (: 1319).

Para finalizar este apartado, dedicado a la relación entre el Capital Educativo familiar y la equidad en el acceso y la participación de los estudiantes en la Universidad española, hemos querido conocer la representación de los estudiantes procedentes de diferentes entornos familiares en la Educación Superior privada.

En el gráfico 5, vemos cómo los estudiantes, cuyos padres poseen estudios medios y altos, se encuentran sobrerrepresentados respecto de la población adulta con igual logro educativo. Son los universitarios, cuyos padres cuentan con estudios superiores, quienes se encuentran fuertemente representados en la Universidad privada (54,43\%) (Véase gráfico 5). Más de la mitad de sus estudiantes pertenecen a este grupo, lo que nos permite concluir que las familias con mayor Capital Educativo tienen una mayor disposición para enviar a sus hijos a este tipo de universidades. En la literatura especializada, se señalan diversas razones para explicar esta tendencia: la mejor información que poseen estas familias del mundo académico y profesional, la reducción del riesgo en contextos de alta competitividad y la búsqueda de distinción académica como mecanismo de reproducción social. 
Por el contrario, los estudiantes cuyos progenitores poseen un nivel educativo bajo, se hayan menos representados que en las públicas. Obviamente, en estos casos, la variable económica juega un papel determinante, dado que, para entrar en este tipo universidades, se requiere pertenecer a entornos económicos con rentas medias-altas y altas, siendo escaso el número de estudiantes procedentes de contextos socioeconómicos bajos que disfrutan de becas en entornos institucionales privados.

\section{Gráfico 5. Estudiantado de universidades privadas según nivel de estudio de los progenitores/ población ambos sexos 40-60 años según nivel de educación 2015-T3}

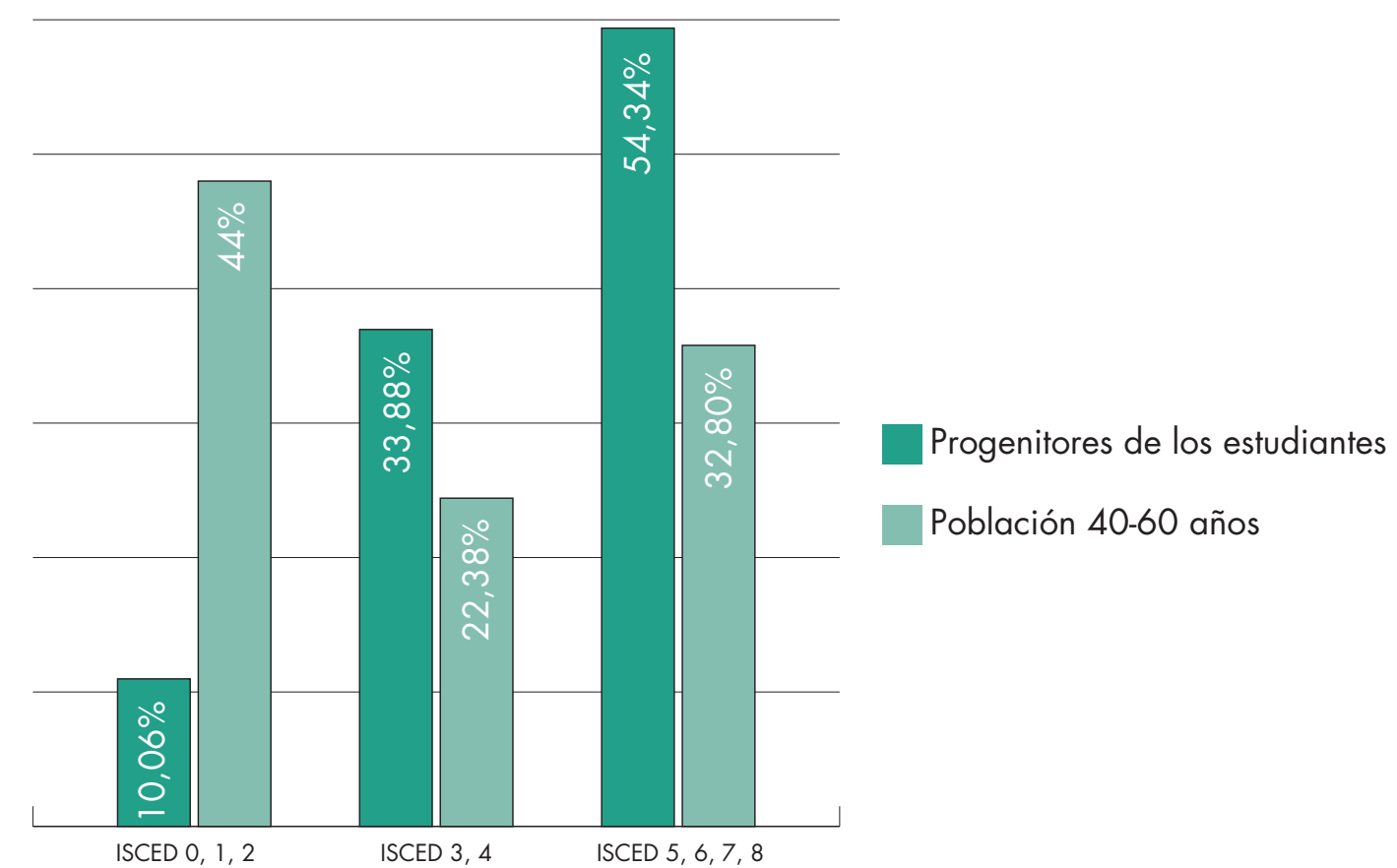

Fuente: elaboración propia a partir de datos estadísticos del MECD.

Estos datos nos llevan a desagregar en nuestro análisis, los dos tipos de universidad coexistentes que configuran el Sistema Universitario en España. Uno, mayoritario, en clara regresión inclusiva a partir del año 2007, debido, en consonancia con otros estudios (Troiano, et al., 2018), a la crisis económica, el incremento de los costes directos de matriculación y los cambios en la política de becas a partir de 2010. Y un segundo tipo de Universidad, en crecimiento, cuyo patrón se acerca al modelo elitista, donde «solo (se) permite el acceso a un pequeño número de personas de una misma clase social y con una elevada preparación académica, que asume además una parte muy importante del coste de la matrícula. Selecciona doblemente: por la capacidad de compra del servicio y por sustentarse en el currículum adquirido en las etapas previas» (Ariño, A., 2014: 37).

\section{Representación del Capital Socioeconómico del estudiantado de nuevo ingreso en el sistema universitario español actual}

En el presente epígrafe, abordamos el análisis sobre el grado de significación del Capital Socioeconómico de los estudiantes y sus familias, en la composición social del sistema universitario para el curso 2015-2016 en España.

En los gráficos 6 y 7 podemos observar cómo los estudiantes cuyo padre ocupa posiciones de «High White collar» (ISCO 1, 2 o 3) y «Low White Collar» (ISCO 4 y 5), presentan una moderada infrarrepre- 
sentación en el conjunto del sistema universitario (29,94\% y 18,53\% respectivamente), en relación con el total de población de ocupados en estas posiciones dentro del mismo grupo de edad (32,86\% y 23,79\%) (Véase Gráfico 6). En el caso de los estudiantes cuya madre se encuentra en alguna de estas posiciones ocupacionales, la infrarrepresentación es algo más pronunciada $(24,47 \%$, ISCO 1, 2, 3, y 22,28\%, ISCO 4 y 5) (Véase gráfico 7).

\section{Gráfico 6. Estudiantado según ocupación del padre/Hombres 40-60 años según ocupación 2015-T3}

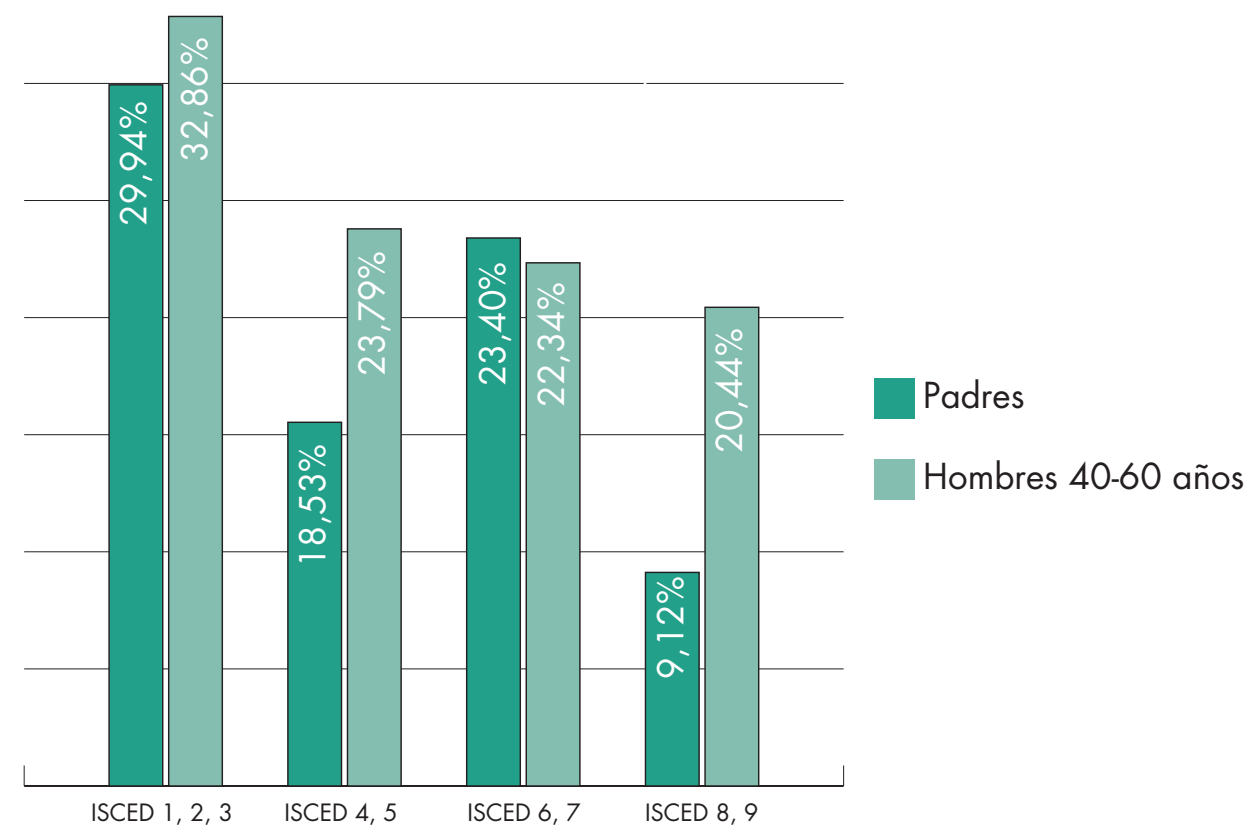

Fuente: elaboración propia a partir de datos estadísticos del MECD y de la EPA.

Tanto en un caso como en el otro, la única categoría ocupacional que se encuentra sobrerrepresentada en el conjunto del sistema universitario, son los estudiantes cuyos padres (23,40\%/22,34\%) (Véase gráfico 6) y, especialmente, las madres (10,81\%/3,31\%), ocupan posiciones ocupacionales de «High Blue Collar» (ISCO 6 y 7) (Véase gráfico 7). Una mayor inversión de estas familias en estudios superiores para sus descendientes encuentra su explicación en la terciarización y globalización de la economía española, dos procesos que vienen afectando directamente a los sectores agrícola e industrial con especial énfasis tras la crisis de 2007.

Finalmente, el grupo social menos representado en la Universidad, son los estudiantes cuyos progenitores se encuentran ocupados en posiciones de «Low Blue Collar» (ISCO 8 y 9). Aquellos cuyos padres se ocupan en estas posiciones, representan apenas el 9,12\%, cuando el grupo de referencia representa el $22,44 \%$ de la población ocupada con edades equivalentes. Mientras que aquellos cuyas madres se ocupan en estas posiciones, apenas representan el 7,62\%, cuando el grupo de referencia representa el 22,06\% (Gráficos 6 y 7, respectivamente). 


\section{Gráfico 7. Estudiantado según ocupación de la madre/Mujeres 40-60 años según ocupación 2015-T3}

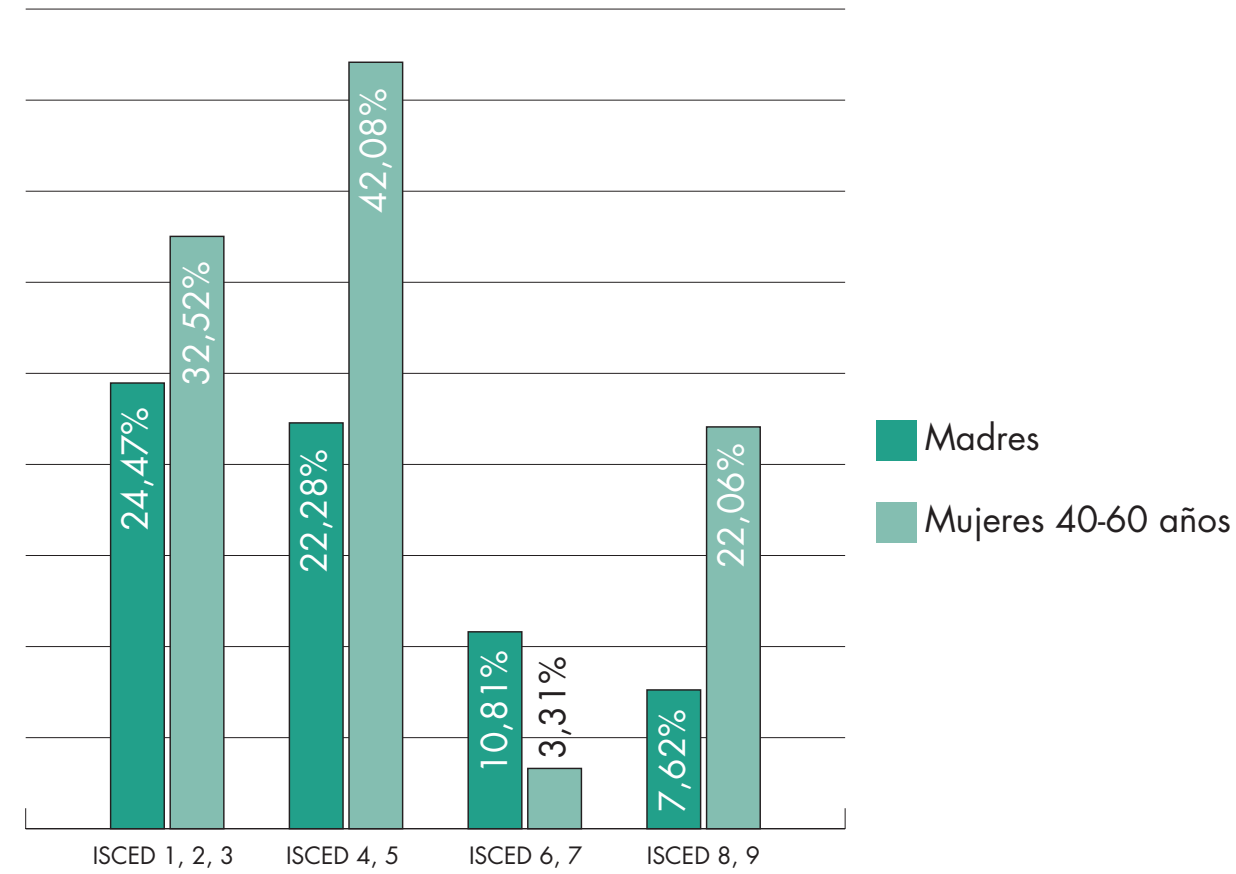

Fuente: elaboración propia a partir de datos estadísticos del MECD y de la EPA.

Estos resultados muestran un empeoramiento de la infrarrepresentación de este colectivo de estudiantes, respecto de los obtenidos cuatro años antes en la encuesta ECoVipeu 2012, donde los estudiantes cuyos padres colocados en estas posiciones representaban el 13,5\%, respecto del conjunto de población de hombres ocupados en estas posiciones (20,1\%). E incluso más fuerte ha sido la caída de los estudiantes cuyas madres se hallaban en esta posición ocupacional. Estos estudiantes representaban en 2011 el $14,06 \%$, cuando el conjunto de población de mujeres ocupadas en estas posiciones era del 23,4\% (Soler, I. 2014: 54). Es decir, entre 2011 y 2016 los estudiantes cuyos padres se encuentran ocupados en posiciones «Low Blue Collar» (ISCO, 8 y 9) cayeron cuatro puntos y medio, y los estudiantes cuyas madres ocupaban estas posiciones redujeron su representación a la mitad, del 14,06\% al 7,62\%.

Los factores causales de la caída de este grupo en tan corto espacio de tiempo podrían coincidir con los descritos en la literatura reciente para el caso de Cataluña, donde se apunta al incremento de los costes directos de la matrícula (Troiano H., \& Torrents, D., 2018), junto a los cambios en las políticas de becas. Los efectos de la crisis, junto al cambio en las condiciones del contexto institucional, sin duda, empujaron a muchas de estas familias a buscar vías alternativas a la Universidad, con mayor motivo si consideramos las restricciones que supusieron las modificaciones introducidas en 2010 y 2013 en el Real Decreto 1.721/2007, de 21 de diciembre, donde se establece el régimen general de becas y ayudas al estudio universitario que concede el MECD. Modificaciones orientadas a aumentar los requisitos de carácter académico exigidos para acceder a una beca (Armenteros et al., 2015:34).

Por último, para concluir este epígrafe, completaremos el estudio descriptivo de la Dimensión Social en la demanda de estudios superiores de la sociedad española, analizando la participación de los estudiantes de diferentes grupos socioeconómicos en las universidades privadas, según la ocupación de los padres.

Dos interpretaciones teóricas tratan de explicar las tendencias en la composición social de la Educación Superior: Maximally Maintained Inequality (MMI) (Raftery, A. E., y Hout M., 1993, Hout, M., 1993) 
y Effectively Maintained Inequality (EMI) (Lucas, 2001). Ambas resultan de gran interés para este trabajo. En la tesis sobre la saturación social escalada en las transiciones educativas planteada por el MMI, se sugiere que, cuando el apoyo público para un determinado nivel de educación cambia, el impacto de los antecedentes sociales sobre la finalización de esa transición educativa también cambiará. Si se reduce, por tanto, el apoyo público a la educación, en nuestro caso a la Educación Superior, los efectos de Clase Social aumentarán (Hout et al., 1993). Por su parte, la EMI postula que los actores socioeconómicamente aventajados obtienen, para ellos y sus hijos, cierto grado de ventaja, cuantitativa o cualitativa, allí donde las condiciones son comúnmente posibles.

La primera de estas interpretaciones resulta, sin duda, un referente explicativo clave para comprender por qué los efectos producidos a partir de los cambios en las condiciones del contexto han tenido como consecuencia una regresión democrática de la composición social en el conjunto del sistema. La segunda puede ayudarnos a comprender las dinámicas estratégicas de las familias con alto Capital Socioeconómico, teniendo presente la, cada vez mayor, importancia que las credenciales exclusivas poseen, en un mercado de títulos convencionales de Educación Superior socialmente saturado. Para ello analizamos, de forma desagregada, la composición social del estudiantado que asiste a la Universidad privada en España.

En los gráficos que siguen (8 y 9), observamos la fuerte sobrerrepresentación que tienen dentro de las universidades privadas, los estudiantes cuyos padres se encuentran situados en las mejores posiciones ocupacionales «High White Collan» (55,85\% sobre 32,86 \%), frente a la moderada infrarrepresentación de los estudiantes de clase «Low White Collar»y «High Blue Collar», (14,54\% sobre 23,79\% y 12,97\% sobre $22,34 \%$, respectivamente).

También, aquellos estudiantes con madres ocupadas en puestos «High White Collar», se encuentran fuertemente sobrerrepresentados en la Universidad privada (41,58\% sobre 32,52\%) (Ver gráfico 9), aunque en una proporción mucho menor que los estudiantes con padres ocupados en la misma categoría $(55,85 \%)$ (Véase gráfico 8$)$.

Los estudiantes cuya madre se ocupa dentro de la categoría «Low White Collar», se encuentran fuertemente infrarrepresentados en la Universidad privada (22,54\%) (Gráfico 9), igual que lo están en la Universidad pública (22,28\%) (Gráfico 7), siendo precisamente esta categoría ocupacional donde mayoritariamente se ocupan las mujeres $(42,08 \%)$.

$\mathrm{Al}$ igual que para todas las universidades, los estudiantes cuyas madres se hallan ocupadas como trabajadoras cualificadas de la industria y del campo (ISCO 6 y 7), también se hallan sobrerrepresentadas en las universidades privadas (6\%) (Véase gráfico 9), si bien en menor medida que en el conjunto de universidades $(10,81 \%$ ) (Véase gráfico 7), lo que refuerza la hipótesis establecida sobre la estrategia de estas familias, empujadas a invertir en educación como respuesta a los cambios derivados de la "globalización" y sus efectos sobre estos sectores económicos. 


\section{Gráfico 8. Estudiantado de universidades privadas según la ocupación del padre/ hombres 40-60 años según ocupación 2015-T3}

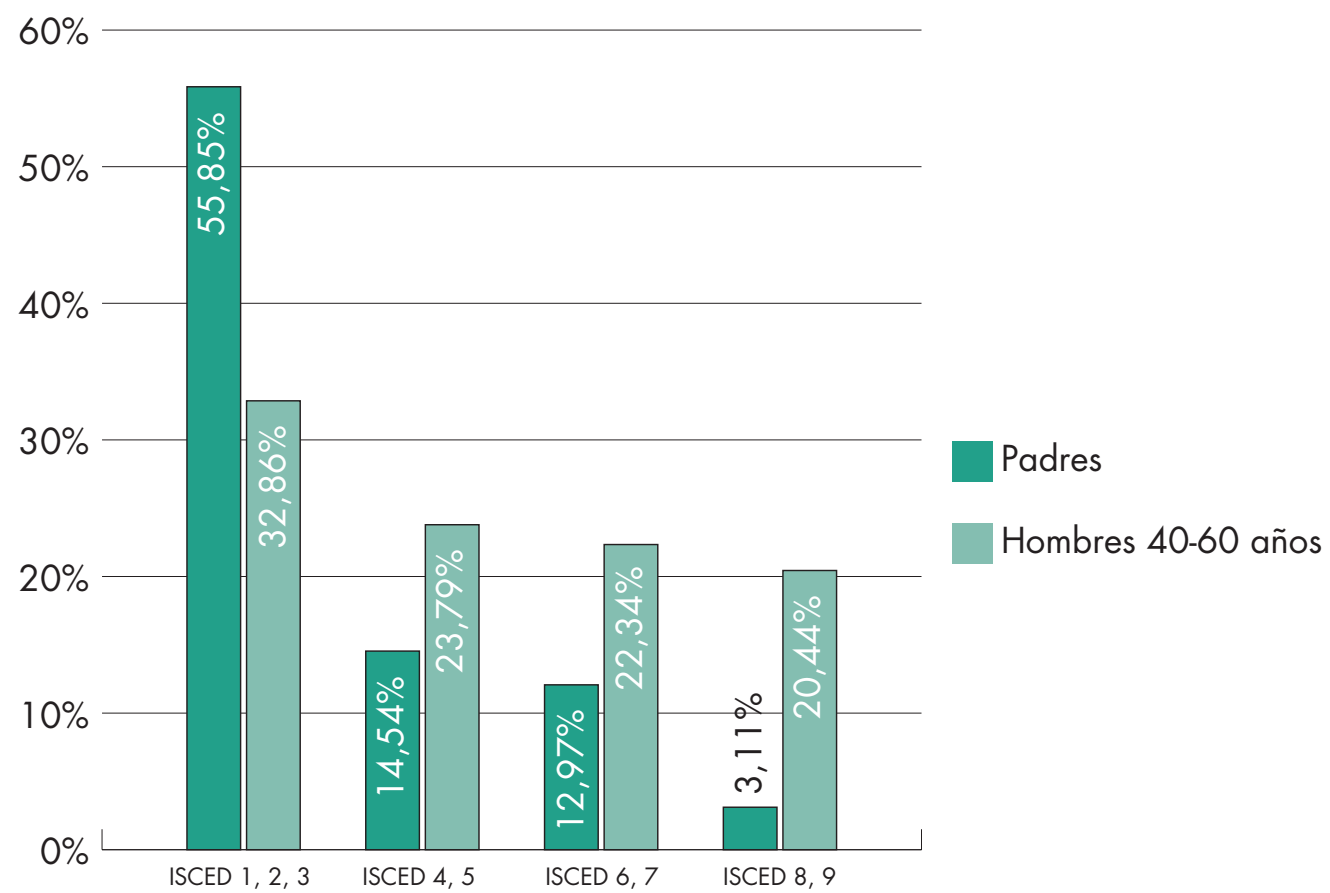

Fuente: elaboración propia a partir de datos estadísticos del MECD y de la EPA.

Por último, los estudiantes cuyos progenitores, cualquiera de los dos, ocupen posiciones «Low Blue Collar», apenas se encuentran representados en la Universidad privada (3\%). La selección por ingresos característica de este tipo de Universidades hace que la representación de este grupo social responda a cifras exiguas de participación en este tipo de Universidad (gráfico 9).

\section{Gráfico 9. Estudiantado de universidades privadas según la ocupación de la madre/ Mujeres 40-60 años según ocupación 2015-T3}

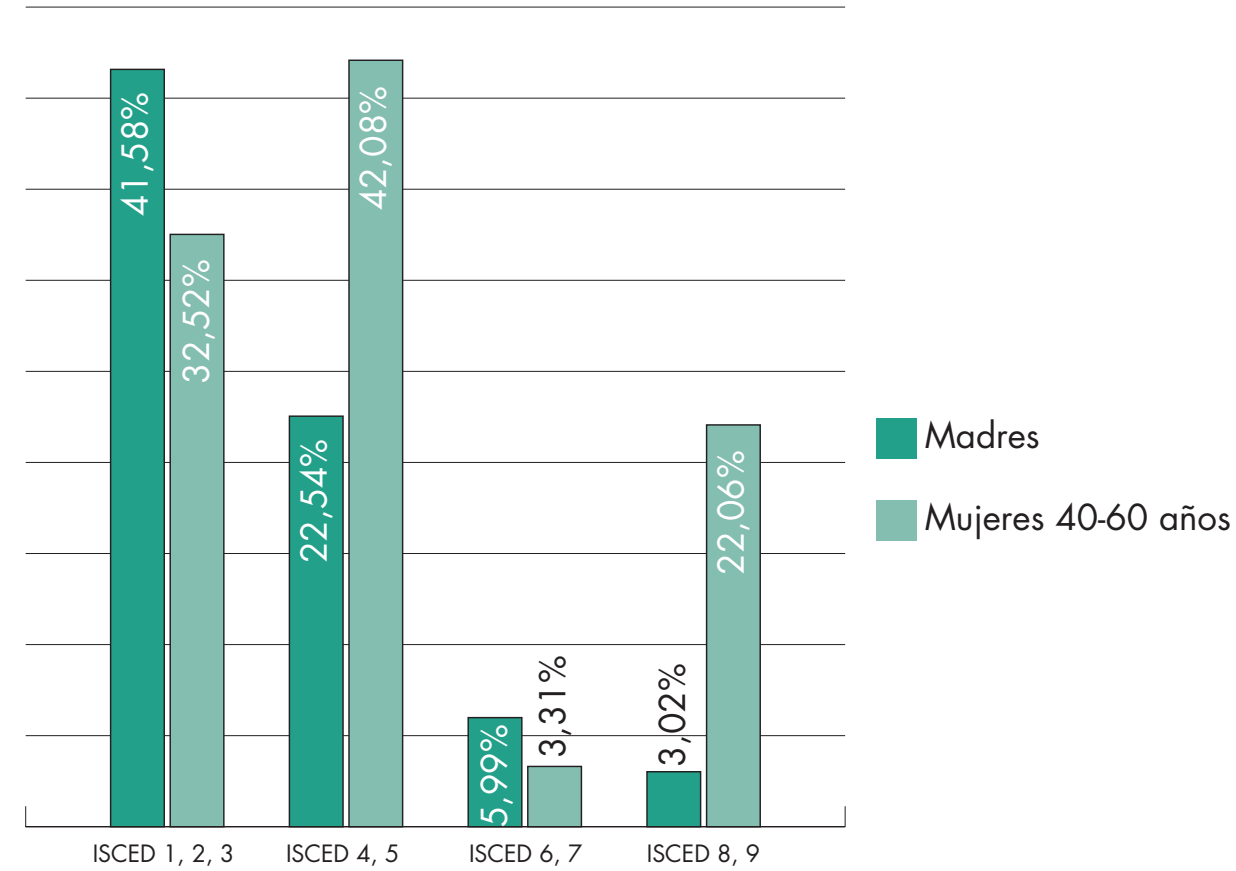

Fuente: elaboración propia a partir de datos estadísticos del MECD y de la EPA. 


\section{Conclusiones}

La primera observación que queremos resaltar de nuestro estudio, es que la movilidad educativa intergeneracional en la sociedad española, en coincidencia con estudios precedentes, mantiene unos niveles altos en la actualidad. En el curso estudiado, algo más del 60\% de estudiantes aspirantes a obtener un título universitario, superarían, en el caso de llegar a lograrlo, el nivel de estudios de sus progenitores.

Partiendo de la idea de que el grado de democratización de la Universidad se evalúa por el grado de participación en ella de los diferentes grupos sociales existentes en la sociedad, la segunda observación importante, es que la democratización en el acceso a la universidad española, si consideramos su composición social en función del Capital Educativo familiar del estudiantado, tal y como se muestra en los gráficos 1 y 2, alcanza un nivel notable en la primera década del siglo xxi, hasta el comienzo de la crisis económica, momento a partir del cual se produce una fuerte regresión democrática de la composición social universitaria.

Con el propósito de profundizar en el conocimiento sobre la evolución de esta tendencia, hemos estudiado la composición social y el grado de equidad participativa de la Universidad española en la actualidad, tomando en consideración, además del Capital Educativo familiar, el Capital Socioeconómico disponible por los estudiantes y sus familias.

Una vez analizados los antecedentes sociales de los estudiantes universitarios, su Capital Socioeconómico y su Capital Educativo, y a la luz de los resultados presentados en este trabajo para el conjunto del sistema universitario, podemos afirmar la existencia de una segregación socioeconómica persistente en el ingreso a la universidad de los jóvenes españoles en el periodo estudiado, curso 2015/2016, periodo en el que se detecta una fuerte caída en la matrícula de estudiantes con un bajo nivel socioeconómico, respecto a los resultados obtenidos por ECoVipeu (2012) para el año 2011. Caída que ha vuelto a alejar a la Universidad española del grado de democratización que había alcanzado en los últimos años del siglo xx y primeros del xxi.

Los resultados obtenidos evidencian la existencia de una infrarrepresentación severa, no solo del estudiantado con bajo Capital Educativo (23,88 \% y 21,45\% según sea el padre o la madre, respectivamente, quien tenga un nivel educativo bajo (ISCED 0,1,2,) (Ver gráficos 3 y 4), también, e incluso mayor, del estudiantado con bajo Capital Socioeconómico (9,12\% y 7,62\%, según sea el padre o la madre, respectivamente, quien se ocupe como Trabajadores no cualificados o Trabajadores empleados en ocupaciones elementales) (Ver gráficos 6 y 7).

Por último, el análisis desagregado de la composición social del estudiantado de la universidad privada presenta a su vez una fuerte sobrerrepresentación de estudiantes con alto Capital Socioeconómico, especialmente de los estudiantes cuyo padre se ocupa en posiciones «High White collar» (55,85\%) (Gráfico 8), y de aquellos estudiantes con alto Capital Educativo (54,43\%, ISCED 5, 6, 7, 8) (Gráfico 5). A partir de estos resultados, podemos decir que la Universidad privada, en la que los estudiantes de las clases populares apenas se encuentran representados, tiende hacia el modelo elitista, el cual, caracterizado por una doble capacidad selectiva, por ingresos y currículum previo, contribuye a aumentar las brechas de desigualdad en los niveles superiores del sistema educativo en España. 


\section{Referencias bibliográficas}

Villarroya Ariño, Antonio (2011): "La Dimensión Social y la participación universitaria” en Antonio Ariño Villarroya y Ramón Llopis: ¿ Universidad sin clases?: condiciones de vida de los estudiantes universitarios en España (Eurostudent IV). Madrid. Subdirección General de Documentación y Publicaciones.

Villarroya Ariño, Antonio (2014): “La Dimensión Social en la Educación Superior”. Revista de la Asociación de Sociología de la Educación (RASE), 7 (1), 17-41.

Villarroya Ariño, Antonio y Llopis, Ramón (2011a). “¿Universidad sin clases? Condiciones de vida de los estudiantes universitarios en España (Eurostudent IV)”. Madrid: Ministerio de Educación.

Villarroya Ariño, Antonio; Llopis, Ramón y Soler, Inés (2014): “Desigualdad y Universidad. La Encuesta de Condiciones de Vida y de Participación de los Estudiantes Universitarios en España. Valencia, Universitat de València". Recuperado: https://www.academia.edu/3638423/Desigualdad_diversidad_y_ Universidad

Armenteros Hernández, Juan y Pérez José Antonio (2017): "La universidad española en cifras 20152016”. Madrid: CRUE Universidades Españolas. [Online]. Recuperado: https://goo.gl/U1zERq

Bertelsmann, Stiftung (2017): "Social Policy in the EU - Reform Barometer 2016, Social Inclusion Monitor Europe, Gütersloh”. Recuperado de: https://www.eurashe.eu/library/modernising-phe/BSt_ SIM_Reform-Barometer2016_WEB.PDF

Carabaña Morales, Julio (1983): “Homogamia y movilidad social”. Reis, 21, 61-81.

Carabaña Morales, Julio y Rivière, Jaime (2017): “¿La igualdad de oportunidades era esto? Estratificación, educación, desigualdad y pobreza. Conversación con Julio Carabaña”. Encrucijadas-Revista Crítica de Ciencias Sociales, 14, 1401.

Comisión Europea (2010a): Europa 2020. Una estrategia para un crecimiento inteligente, sostenible e integrador, COM (2010) 2020. Bruselas. Recuperado de: https:/ / ec.europa.eu/info/business-economy-euro/economic-and-fiscal-policy-coordination/eu-economic-governance-monitoring-prevention-correction/ european-semester/framework/europe-2020-strategy_es.

Comisión Europea/EACEA/Eurydice (2015): El Espacio Europeo de Educación Superior en 2015: Informe sobre la implantación del Proceso de Bolonia. Luxemburgo: Oficina de Publicaciones de la Unión Europea. Recuperado de: http://publications.europa.eu/resource/cellar/91f926b2-6965-4abe-a1be600903e4df93.0003.01/DOC_1.

Conclusiones del Consejo del 11 de mayo 2010, sobre la Dimensión Social de la educación y la formación, O JC 135, 26.05.2010. Recuperado de: https:// eur-lex.europa.eu/LexUriServ/LexUriServ.do?u ri=OJ:C:2010:135:0002:0007:ES:PDF.

Consejo Europeo (2017, 17 noviembre): Pilar europeo de derechos sociales: proclamación y firma - Consilium [Comunicado de prensa]. Recuperado 28 septiembre, 2018, de http://www.consilium.europa.eu/es/ press/press-releases/2017/11/17/european-pillar-of-social-rights-proclamation-and-signing/.

Fachelli, Sandra y Planas, Jordi (2011): "Equidad y movilidad intergeneracional de los titulados universitarios catalanes”. Papers: revista de sociología, 96 (4), 1307-1331. 
Hout, Michael (2006): "Maximally Maintained Inequality and Essentially Maintained Inequality". Sociological Theory and Methods, 21 (2), 237-252.

Instituto Nacional de Estadística (INE) Clasificación Nacional de Ocupaciones (CNO-11). Recuperado: Clasificación Nacional de Ocupaciones (CNO-11).

Instituto Nacional de Estadística (INE). Encuesta de población activa (EPA) Recuperado de: http:// www.ine.es/dyngs/inebase/es/operacion.htm?C=Estadistica_C\&cid=1254736176918\&menu=ultid atos\&idp $=1254735976595$.

Instituto Nacional de Estadística (INE), 2016T4. Recuperado de: http://www.ine.es/jaxiT3/Datos. $h \mathrm{tm} ? \mathrm{t}=6393$

Jerrim, John; Vignoles, Anna; Lingam, Raghu y Friend, Angela (2014): “The Socio-Economic Gradient in Children's Reading Skills and the Role of Genetics”. British Educational Research Journal, 41 (1), 6-29.

Lucas, Samuel (2001): "Effectively maintained inequality: Education transitions, track mobility, and social background effects". American Journal of Sociology, 106 (6), 1642-1690.

Ministerio de Educación, Cultura y Deporte. Estadísticas universitarias. Entrada al sistema universitario. Acceso a la Universidad 2017. Última consulta julio de 2018: https://www.educacion.gob.es/educabase $/$ menu.do?'Type=pcaxis\&path=/Universitaria/Indicadores $/ 2017 / 1 \_$Acceso\&file=pcaxis\&l=s0.

Ministros de Educación Superior. (2007): Comunicado de Londres. Hacia el Espacio Europeo de Educación Superior: respondiendo a los retos de un mundo globalizado. Recuperado de: http://www. aneca.es/Actividad-internacional/Documentos-internacionales-de-referencia/Comunicados-de-losministros-europeos.

Nielsen, François y Roos, J. Micah (2015): "Genetics of Educational Attainment and the Persistence of Privilege at the Turn of the $21^{\text {st }}$ Century”. Social Forces, 94 (2), 535-561.

OECD (2018). Equity in Education: Breaking Down Barriers to Social Mobility. PISA: OECD Publishing, Paris. https://doi.org/10.1787/9789264073234-en.

Soler, Inés (2014): "Perfil social y bagaje familiar", en Antonio Ariño Villarroya, Ramón Llopis e Inés Sóler: Desigualdad y Universidad. La Encuesta de Condiciones de Vida y de Participación de los Estudiantes Universitarios en España. Valencia: Universitat de València. España: EcoVIPEU.

Raftery, Adrian E. y Hout, Michael (1993): "Maximally Maintained Inequality: Expansion, Reform, and Opportunity in Irish Education, 1921-75”. Sociology of Education, 41-62.

Rahona, Marta Mercedes (2008). La educación universitaria en España y la inserción laboral de los graduados en la década de los noventa. Un enfoque comparado. Madrid: Premios Injuve para Tesis Doctoral.

Troaino, Helena y Torrents, Dani (2018). "La evolución del acceso a la universidad en Catalunya: ¿cómo la explicamos?”. Revista Española de Sociología, 27 (1), 125-134).

\section{Nota biográfica}

Damián Herrera Cuesta eDamián Herrera Cuesta es Doctor en Sociología (UNED), realizó el Máster en Análisis de Problemas Sociales en el Departamento de Sociología III de la Facultad de Ciencias Polí- 
ticas y Sociología de la UNED, donde ha sido Personal Docente e Investigador. Ejerce como consultor e investigador social y ha trabajado como sociólogo en el Ayuntamiento de Oviedo. Ha publicado, recientemente, el artículo: "Empleabilidad versus sobrecualificación. Desajuste entre formación y empleo en las trayectorias laborales de los jóvenes titulados en España”, Sociología del Trabajo, 89 (2017), y el capítulo: “El mercado laboral de los titulados en España. El fenómeno de la sobrecualificación”, en Investigación y prácticas sociológicas: escenarios para la transformación social, UNED, 2018. 